\title{
Cause analysis and clinical management experience of the premature rupture of membrane
}

\author{
Ning Li, Qiulan Fu, Wenhua Cai \\ Department of Obstetrics and Gynecology, Nanning Maternal and Child Health Care Hospital, Nanning, China \\ Email: lining0771@163.com
}

Received 13 December 2012; revised 15 January 2013; accepted 24 January 2013

\begin{abstract}
Based on the retrospective study on 189 cases of premature rupture of membrane maternity, we find that PROM can result in higher risks of dystocia, cesarean birth and maternal and fetal complications, upon examining our clinical management measures and searching for a positive clinical management, we are looking for a better way to reduce PROM and the risk of mother and child, to have a better pregnancy outcome.
\end{abstract}

Keywords: PROM; Dystocia; Puerperal Infection; Neonatal Suffocation; Neonatal Pneumonia

\section{INTRODUCTION}

Premature rupture of membrane (PROM) is a common obstetric complication, which is divided into term PROM and preterm PROM clinically [1]. 90\% of the PROM patients will experience large amount of liquid flowing out from vaginal, with no sign of abdominal pain or other production omen. When pushing up fetal presentation department, liquid can be seen flowing out from vagina and fetal fat can be detected in the effluent sometimes. Yellow-green effluent can be seen when fetus experience intrauterine distress, which can lead to the amniotic fluid pollution caused by meconium. If obvious amniotic cavity infection occurs, the vaginal effluent smells, associated with acute infection performance such as maternal fever, mother \& child's heart rate increasing, pressing pain in uterine, etc. When concurrent stealthiness amniotic cavity infection occurs, the clinical manifestation of increasing heart rate of mother \& child often happens although no clinical observation to parents with obvious fever. Uterine contraction and dilatation of cervix will often occur shortly after having PROM or PPROM. Study [2] shows that PROM is closely related to vaginal infection and it is the beginning of the dystocia as it often leads to amniotic fluid reduction and uterine inertia, etc., which will easily cause dystocia and surgical delivery.
PROM can also cause premature delivery, circumference begat infection, which are the main causes of stillbirth, fetal distress and neonatal asphyxia, neonatal pneumonia [3].

\section{OBJECT AND METHOD}

\subsection{The Research Object}

The authors chose 189 cases of pregnant women diagnosed with premature rupture of membrane in Nanning Maternal and Child Health Care Center from June 2009September 2010 as observation group and 864 healthy pregnant women who delivered without PROM in the same period as control group. The women in observation group are aged from 20 to 35 , with average age of ( 28 $+/-3.05)$, gestational weeks from 27 to 41 weeks, with the average gestational weeks of $(38+/-2.07)$. The women in control group are aged from 22 to 39 , with an average age of $(27.5+/-4.72)$, gestational weeks from 28 to 40 weeks, with the average gestational weeks of $(39+/-0.44)$. There is no statistical significance on age and gestational weeks between the two groups ( $\mathrm{P}>$ $0.05)$.

\subsection{Methods}

1) Diagnosis method

a) Liquid flows out from vagina or large amount of fetal fat and meconium can be seen in vagina dome when expanding vagina with vaginal speculum.

b) $\mathrm{pH}$ value $>6.5$ when measured vaginal effluent with Litmus paper.

c) Fetal skin from the epithelial cells and amniotic fluid crystallization found in vaginal discharge fluid smear.

d) Amniotic fluid flows out upon pushing up the presenting part.

2) Processing method

Vaginal secretions mycoplasma from the observation group is cultivated to observe combined amniotic cavity infection. If it is infected, terminates pregnancy; if not, 
they will be told to rest in beds, with recumbent position or lateral position on bed with raised tail. Keep the vulva clean and avoid vaginal examination and anal check. Exercise fetal electronic monitoring regularly, make biological, physical score to determine fetal intrauterine condition if necessary. Regularly examine by B ultrasound, record the amount of amniotic fluid, fetal growth and development. Inject with $5 \mathrm{mg}$ dexamethasone intramuscular, two times a day, a total of $2 \mathrm{D}$, promoting lung maturation. If gestational weeks $>35$ weeks, observe for $12 \mathrm{~h}$, and antibiotics will be used if there is no sign of laboring; If childbirth does not happen $24 \mathrm{~h}$ later, use intravenous drip of oxytocin or prostaglandin E2 bolt (trade name: Dinoprostone Suppositories) to induce labor. Neonates Apgar score $\leq 7$ are judged as neonate asphyxia.

\subsection{Statistical Processing}

SPSS17.0 statistical software is used. $\mathrm{x} \pm \mathrm{s}$ is applied to measurement data, and the two groups are compared with $\mathrm{t}$ test. Numeration data are expressed in percentage. The two groups are compared by using $\chi^{2}$ test. If $\mathrm{P}<$ 0.05 , the difference is statistically significant.

\section{RESULT}

\subsection{The Incidence Rate of PROM}

There were PROM 189 cases in 1053 delivery cases in Nanning Maternal and child health hospital from June 1, 2009 to September 30, 2010, accounting for $17.9 \%$.

\subsection{The Relationship between PROM and Genital Tract Infection}

In the observation group, 57 cases $(30.2 \%)$ are positive in vagina secreta bacertial culture; in the control group, 93 cases $(10.76 \%)$ are positive in vagina secreta bacertial culture. The difference between the two groups is statistically significant $(\mathrm{P}<0.05)$.

\subsection{Parturient Fetal Head Articulations}

In the observation group, the cases of fetal head articulation and without articulation are $67(35.4 \%)$ and 122 $(64.6 \%)$ respectively, in the control group are 645 cases
(74.7\%) and 219 cases $(25.3 \%)$ respectively. The difference between the two groups are statistically significant $(\mathrm{P}<0.05)$.

\subsection{Mode of Delivery}

The observation group natural delivery rate is significantly lower than the control group $(\mathrm{P}<0.01)$. Cephalopelvic disproportion, fetal distress rate is significantly lower $(\mathrm{P}<0.01)$, see Table 1.

\subsection{Maternal and Fetal Complications}

1) Neonatal premature delivery. 34 cases of premature $(18 \%)$ in the observation group, 57 cases in the control group $(6.6 \%)$, the two groups are statistically significant $(\mathrm{P}<0.05)$. The average gestational weeks of preterm infants in the observation group are $(34 \pm 1.72)$ weeks, average weight of $(2.4 \pm 0.24) \mathrm{kg}$; Those in control group are $(35 \pm 0.98)$ weeks, $(2.5 \pm 0.13) \mathrm{kg}$. The difference is not significant $(\mathrm{P}>0.05)$.

2) Neonatal complications. There are 45 cases $(23.8 \%)$ of neonatal asphyxia in observation group, 110 cases $(12.7 \%)$ in the control group; 31 cases with neonatal pneumonia (16.4\%) in observation group, 77 cases $(8.9 \%)$ in control group. The group difference of the two cases is statistically significant $(\mathrm{P}<0.05)$.

3) Maternal and fetal complications (puerperal infection). There are 5 cases of puerperal infection $(2.64 \%)$ in observation group, 4 cases in control group $(0.46 \%)$. The difference between the two groups is statistically significant $(\mathrm{P}<0.05)$.

\section{DISCUSSION}

PROM is a common complication of pregnancy. Infection, preterm birth, placental abruption, prolapse of umbilical cord, amniotic fluid, fetal distress and reduced complication rates are significantly increased because of PROM, which can often lead to adverse pregnancy outcome. Current clinical treatment for PROM, especially PPROM, has been progressed, but there is no ideal method yet. Therefore, the research on the cause of PROM, as well as how to treat PROM and prevent other pregnancy complications is of great significance for obstetric clinical diagnosis and treatment.

Table 1. Comparison of delivery modes in two groups.

\begin{tabular}{|c|c|c|c|c|c|c|c|c|c|c|c|}
\hline \multirow{3}{*}{$\begin{array}{c}\text { Goups } \\
\text { Observation }\end{array}$} & \multirow{3}{*}{$\begin{array}{c}\mathrm{N} \\
189\end{array}$} & \multicolumn{4}{|c|}{ Vaginal Delivery } & \multicolumn{6}{|c|}{ Cesarean Birth } \\
\hline & & \multicolumn{2}{|c|}{ Natural Birth } & \multicolumn{2}{|c|}{ Assisted Delivery } & \multicolumn{2}{|c|}{ Cephalopelvic Disproportion } & \multicolumn{2}{|c|}{ Breech Position } & \multicolumn{2}{|c|}{ Fetal Distress } \\
\hline & & 92 & $(48.7)^{*}$ & 1 & $(0.5)$ & 42 & $(22.2)^{*}$ & 14 & (7.4) & 40 & $(21.2)^{*}$ \\
\hline Control & 864 & 621 & (71.9) & 4 & $(0.5)$ & 121 & $(14.0)$ & 6 & $(0.7)$ & 112 & $(13.0)$ \\
\hline
\end{tabular}

$[\mathrm{n}(\%)]$; Compared with the control group: ${ }^{*} \mathrm{P}<0.01$. 


\subsection{The Risk Factors for PROM}

There are many risk factors for PROM. In most cases, the various risk factors coexist and interact which as a result cause PROM. Here are two major risk factors:

1) Genital tract pathogenic microorganism ascending infection. Ziaei S cultivated 200 PROM patients of vaginal discharge and uterine secretions and compared with the control groups. He believed that PROM is related with vaginal infection [4] Aboyeji AP drew the conclusion from the statistics of the inspection results of PROM vaginal secretions that PROM is closely related to vaginal infections [5].

Our research shows that the vaginal bacterial culture positive rate is $30.2 \%(57 / 189)$ in 189 cases of PROM in pregnant women, while the vagina secreta bacertial culture positive rate is $10.76 \%(93 / 864)$ in control group. The genital tract infection rate is significantly higher than that of control group $(\mathrm{P}<0.05)$, thus the fetal membrane rupture and reproductive tract infections are highly associated.

2) Elevated pressure on Amniotic cavity. Twin pregnancy and polyhydramnios can increase amniotic cavity pressure. When companying with fetal membrane defects, such as the loss of elasticity, collagen reduction, the increasing pressure acting on the weak fetal membrane place will cause premature rupture of membrane.

3) Uneven pressure on fetal membrane. Such abnormal conditions as abnormal fetal position, asymmetric head basin etc. can cause the failure on engagement between the fetal presentation department and the pelvic entrance. The empty pelvic can result in uneven pressure on the front amniotic fluid capsule, causing premature rupture of membrane.

4) Insufficient nutrients. Study proves that the lack of vitamin $\mathrm{C}$ and cuprum in mother blood can reduce the tensile ability of fetal membrane, which can easily cause premature rupture of membrane.

5) Dilated cervix. Factors such as surgery mechanical expansion on cervix, birth trauma or congenital cervical vulnerable structure can damage the function of cervix musculi sphincter. The consequence is that the cervical mouth will loosen, which makes it the place the front amniotic fluid capsule can easily wedge into, causing uneven pressure on the amniotic fluid capsule. This part of fetal membrane is close to the vagina, lacking of cervical mucus protection, so it is likely to be infected by pathogenic microorganism, which will cause premature rupture of membrane.

\subsection{The Relationship between PROM and Dystocia}

The observation of 189 cases of pregnant women shows that the rate of fetal heads without articulation is $64.6 \%$
(122/189), higher than that of control group, 25.3\% (219/ 864 two). The difference is statistically significant $(\mathrm{P}<$ 0.05 ). The data shows that the main reason for cesarean birth (dystocia) lies in the cephalopelvic disproportion, incorrect fetal position (breech) and fetal distress these three aspects.

The details are as follows: the cesarean birth rate caused by disproportion is $22.2 \%(42 / 189)$ in observation group, $14 \%(121 / 864)$ in control group; the cesarean birth rate caused by breech position is $7.4 \%(14 / 189)$ in observation group, $0.7 \%(6 / 864)$ in control group; the cesarean birth rate caused by fetal distress is $21.2 \%$ $(40 / 189)$ in observation group, $13 \%(112 / 864)$ in control group. the difference between the two groups in three circumstance above is statistically significant $(\mathrm{P}<0.05)$. It means that there is relation between PROM and dystocia.

The reason is that when in the cephalopelvic disproportion or abnormal fetal position, fetal presentation and pelvic floor can not engage properly, which will leave gaps between fetal presentation and pelvis. When the uterine pressure rises, increased intrauterine pressure is applied to the amniotic sac evenly through the gaps, which can lead to premature rupture of membrane, amniotic fluid decreasing quickly and amniotic fluid cushioning effect reducing dramatically. As a consquence, umbilical cord will be sequeezed by sterine wall and foetus, causing cord blood circulation disorder and fetal distress, increasing the chance of cesarean birth. Therefore, premature rupture of membranes often indicates dystocia.

\subsection{PROM Effects on the Mother and Infant}

The mother of puerperal infection is one of the main complications of PROM. Data in this article shows that, the puerperal infection rate is $2.64 \%(5 / 189)$ in observation group, $0.46 \%(4 / 864)$ in control group. The difference between the two groups is statistically significant ( $\mathrm{P}$ $<0.05$ ). The reason is that PROM can cause pathogen retrograde, chorioamnionitis, endometritis, and operation incision infection etc. It can also cause fatal placental abruption, severe uterine empyema, pelvic inflammatory disease, such as peritonitis, sepsis, and septic shock and other serious complications, causing maternal severe infection, even death. PROM can normally cause premature preterm birth, $30 \%-50 \%$ of which is associated with PROM [6].

PROM can also result in the reduction of amniotic fluid volume which can cause intrauterine fetal distress, leading to perinatal infections, complications, fetal umbilical cord compression syndrome etc, which make the morbidity of fetal distress, neonatal asphyxia and neonatal pneumonia increase significantly [7]. 
The data shows that between the two groups with which there is no statistically significant difference on fetal gestational age and body weight the preterm birth rate is $18 \%(34 / 189)$ in observation group, the control group is $6.6 \%(57 / 864)$.

The data also shows that the neonatal asphyxia rate in observation group is $23.8 \%(45 / 189)$, control group is $12.7 \%(110 / 864)$; neonatal pneumonia $16.4 \%$ in observation groups (31/189), the control group 8.9\% (77/864). They are of statistically significant difference (mean value of $\mathrm{P}<0.05$ ). So PROM would increase the possibility of preterm delivery and complications of prematurity.

\subsection{The Prevention of PROM}

The five risk factors mentioned above often interact and occur together or partially, so we should take action accordingly to prevent PROM. The main prevention methods can be:

1) Lower genital tract infection, such as richomonas vaginitis, gonorrhea nye's bacteria infection, cervical chlamydia trachomatis infection, bacterial vaginal disease, etc., should be treated as early as possible to prevent PROM.

2) Pay attention to nutrition balance, appropriate supplementary copper element or vitamin $C$.

3) Avoid sudden increase of abdominal pressure. Take enough rest for patients who are experiencing high floating presentation of feta, excessive uterus expansion.

4) For cervical dilation, carry out cervical cerclage on 14 - 16 weeks in pregnancy.

\subsection{The Treatment of PROM}

We fully informed PROM patients and their families the possible risks and complications in the progress of laboring and trial-produce process, carried out clinical management and treatment in the condition that patients have signed the consent for treatment. The management and treatment measures have been approved by our ethics and academic committee, and have become obstetric medical treatment standard for the obstetrical department. The following are our clinical management measures on pregnant women with premature rupture of membranes.

1) Expectant treatment. For pregnant women who are not in labor after rupture of membranes, and with no signs of infection, gestational weeks in 28 - 34 weeks, B ultrasound showing amniotic fluid pool depth $\geq 3 \mathrm{~cm}$, expectant treatment can be applied.

Processing measures are as follows:

a) Bed rest, avoid unnecessary anal and vaginal examination, maintain vulva clean, test body temperature, pulse, the characteristics of the amniotic fluid and smell regularly. Check amount of leukocyte and neutrophil classification daily for 3 consecutive days, if normal, then 2 times a week, beware of chorioamnionitis.

b) Use antibiotics if the film has broken for more than 12 hours.

c) Uterine contraction will happen within 24 hours on most pregnant women with premature rupture of membranes. To extend gestational age, tocolytic agents should be used according to the situation.

d) To accelerate the fetal lung maturation, use dexamethasone $5 \mathrm{mg}$ by intramuscular injection every 12 hours, 4 times in total.

e) Monitor the residual volume of amniotic fluid with B ultrasound, if the depth of the amniotic fluid pool $\leq 5$ $\mathrm{cm}$, drink $2000 \mathrm{ml}$ water within 2 hours, which can increase the amount of amniotic fluid. If the amniotic fluid pool depth $\leq 2 \mathrm{~cm}$, consider termination of pregnancy.

f) The fetal heart rate monitoring and $\mathrm{C}$ reactive protein examination can diagnose chorioamnionitis. When $\mathrm{C}$ reaction protein $>30 \mathrm{mg}$, consider the presence of infection, once diagnosed, terminate pregnancy.

2) The time for termination of pregnancy. If labor happens after 35 weeks of pregnancy, let it go; For those who have the indication of cesarean, terminate pregnancy by cesarean; As for the women whose pregnancy with diabetes are not up to 36 weeks, promote fetal lung maturity on the condition of normal amniotic fluid index and no infection, and extend the weeks of gestation to over 36 weeks.

3) PROM parturient management. Close observation on fetal presentation articulation, fetal heart sound, uterine contractions, and characteristics of the amniotic fluid. Handle the first and second birth process actively, shorten the production process to reduce the incidence of neonatal asphyxia.

If the labor progress is slow, identify the reasons, use oxytocin, uterine cervix, lidocaine and cervical dilation with hand to shorten the birth process. Try trial production with the monitoring of continuous fetal heart rate. If vaginal delivery is not possible, choose cesarean delivery without delay.

4) Postpartum treatment. After giving birth, bacterial cultivation and drug sensitive test should be used for pharynx, ear canal and skin, and pathological examination on placenta, fetal membranes; Intensive observation on mother and infant about any sign of infection.

5) Prevent from maternal postpartum puerperal Infection. The specific method: preoperative use of adequate amounts of antibiotics; flush the uterine cavity and the operation incision with metronidazole during operation; use antibiotics 5 - 7 days after operation; pay attention to perineal care to avoid repeated infection; Pull out catheter as early as possible, make patients drink more water to avoid urinary tract infection; douche vaginal with iodophors after maternal third production process for vaginal delivery. 
After taking the above the prevention and treatment measures, we have reduced the puerperal infection rates of PROM patients, prolonged the fetal gestational weeks, used antibiotics more efficiently, have been more appropriate on the timing of surgery cesarean section, have been more standard and positive on treatments to patients with PROM in prenatal, intrapartum, postpartum, and also, we have reduced the PROM neonatal complications. All these have had a positive effect on PROM maternal postpartum psychological and physiological recovery.

PROM is closely related to genital tract pathogenic microorganism ascending infection, amniotic cavity elevated pressure, uneven stress on fetal membrane field insufficient nutrients, and cervical dilation, among which genital tract pathogenic microorganism ascending infection is the most common one. Premature rupture of membrane often indicates the occurrence of dystocia, hence increases the chances of surgery. After the occurrence of PROM, the incidence rate of complications such as premature babies, neonatal suffocation, neonatal pneumonia, maternal puerperal infection will increase significantly, resulting in adverse pregnancy outcome. So upon preventing PROM, the first thing should be popularizing the knowledge of healthy pregnancy, carrying out prenatal care properly, especially paying attention to the screening and treatment of the lower genital tract infection of pathogenic microorganisms.

Use expectant therapy to prolong gestational age once preterm PROM is detected. Terminate pregnancy in the event of fetal mature or infection syndrome [8]. If no cephalopelvic disproportion, oxytocin should be used; If cephalopelvic disproportion, fetal distress and other indications for cesarean birth, terminate pregnancy so as to reduce the incidence of complications, prepare for the clinical management of PROM.

\section{REFERENCES}

[1] Le, J., Xie, X. and Feng, A. (2004) Obstetrics and gynecology. 6th Edition, People's Medical Publishing House, Beijing, 145-146.

[2] Zhang, S.H. and Li, H.F. (2008) Clinical analysis of 130 cases of fetal membranes dystocia caused by premature rupture. Shannxi Medical Journal, 37, 1006-1007.

[3] Yang, W., Wan, H.P. and Lan, F.F. (2009) Social psychosocial factors analysis on the influence of treatment of preterm premature rupture of membranes. Modern Preventive Medicine, 36, 3693-3694.

[4] Ziaei, S., Sadrkhanlu, M., Moeini, A., et al. (2006) Effect of bacterial vaginosis on premature rupture of membranes and related complications in pregnant women with a gestational age of 37 - 42 weeks. Gynecology and Obstetrics Investigation, 61, 135-138. doi:10.1159/000090086

[5] Aboyeji, A.P., Abdul, I.F., Ijaiya, M.A., et al. (2005) The bacteriology of pre-labour rupture of membranes in a $\mathrm{Ni}$ gerian teaching hospital. Journal of Obstetrics and Gynecology, 25, 761-764.

[6] Aman, C. (2010) End analysis on preterm premature rupture of membranes premature. Chinese Community Physicians, 12, 50-51.

[7] Tiufekchieva, E. (2006) Intrauterine infection in cases with premature rupture of fetal membranes-incidence, structure. Akusherstvo I Ginekologiia, 45, 9-15.

[8] Li, C.X., Xu, J.P. and Yin J.F. (2009) A clinical analysis of 162 cases of premature rupture of membranes. Maternal and Child Health Care of China, 24, 1299. 